\title{
Influence of Calcification Solution on in vitro Chitosan
}

\section{Mineralization}

\author{
Marisa M. Верpu*, Cesar C. Santana \\ Faculdade de Engenharia Química, Unicamp, \\ C.P. 6066 13081-000 Campinas - SP, Brazil
}

Received: November 14, 2000; Revised: January 10, 2002

\begin{abstract}
The influence of the calcification solution on the mineralization of chitosan membranes was analysed. Two factors were studied: $\mathrm{pH}$ and chemical composition (excess of calcium or phosphate ions) of solutions. Substrates (membranes) of chitosan underwent mineralization by soaking in calcification solutions for 7 days at $36.5^{\circ} \mathrm{C}$. As expected, SEM-EDS analyses demonstrated that higher $\mathrm{pH}$ and concentration of ions increases deposition, however, the morphology and composition of these deposits varies depending on which ion (phosphate or calcium) is in excess. Larger spherical deposits are observed when a calcium-rich solution is used instead of a phosphate-rich one. In the latter, the interconnectivity of the inorganic and organic (matrix) phases is very different, suggesting a different mechanism of deposition for each situation.
\end{abstract}

Keywords: chitosan, chitin, calcification, mineralization

\section{Introduction}

Calcification or mineralization can be defined as the formation of calcium phosphate or other calcium compounds. In mineralization, these inorganic deposits are formed with an organic matrix controlling their shape, size and orientation. In situ deposition is an alternative to avoid the usual difficulties found in sol-gel processes, such as particle aggregation.

The mineralization phenomenon has aroused the interest of many scientific and technological areas for a number of reasons. For material scientists, mineralization can produce functional and resistant composites. In medicine, knowledge of mineralization will lead to a better understanding of bone and teeth calcification in order to develop prosthesis and ostheogenic implants or to avoid pathological calcification of organs and medical devices (mainly for cardiac devices) ${ }^{1}$.

Calcification can be desirable, or not, depending on the biomaterial end use. Thus, controlling its occurrence is crucial and requires deep knowledge of the subject.

Chitosan has recently been appointed a biomaterial with significant potential for use in many fields, such as in medical science, biotechnology, food science etc. Composed mainly of 2-amino-2-deoxi-D-glucose. Chitosan has received much attention recently because it is a renewable,

e-mail: *beppu@feq.unicamp.br, ${ }^{\#}$ santana@ feq.unicamp.br Trabalho apresentado no $14^{\circ}$ CBECIMAT, Águas de São Pedro, Dezembro de 2000 . biodegradable and biocompatible material. It can also be shaped into several forms, has amino and hydroxyl groups that can be easily reacted, and is derived from the second most abundant natural polymer worldwide (just loosing to (cellulose $)^{2}$. However, an application that has not been explored, so far, is as an organic matrix for the production of biocomposites using biomimetic processes.

In the present work, we studied the mechanism of in vitro calcification of chitosan extracted from crab shells. Porous chitosan membranes underwent an in vitro calcification process using several calcification solutions in order to verify the influence of their composition and $\mathrm{pH}$ upon the composition and morphology of calcification deposits.

\section{Experimental}

All reagents were of analytical grade. Milli-Q water was used in all solutions.

Porous chitosan membranes were prepared by casting and coagulation as described elsewhere ${ }^{3}$. Chitosan extracted from crab shells (Sigma, St. Louis, MO - product number C 3646, minimum of 85\% deacetylated chitin) was used.

Calcification solutions were prepared by mixing solutions of the following salts: $\mathrm{NaCl}, \mathrm{KCl}, \mathrm{CaCl}_{2}, \mathrm{MgCl}_{2}$, $\mathrm{NaHCO}_{3}, \mathrm{~K}_{2} \mathrm{HPO}_{4}$ and $\mathrm{Na}_{2} \mathrm{SO}_{4}$. Four kinds of solutions 
were produced and their compositions are shown in Table 1. $\mathrm{pH}$ was adjusted with $\mathrm{HCl}$.

In vitro experiments were performed as follows: the substrates ( $2 \times 1 \mathrm{~cm}^{2}$ chitosan membrane pieces) were soaked in a solution ( $\mathrm{pH}-7.4$ to 7.8 ) which simulated 1 (for two days) and 1.5 times (for the remainder of the days) the salt concentration of body fluids (plasma) for a total of 7 days. This protocol was used with the idea that the first days are the most important for the induction of the first deposits. A more concentrated solution was then used to induce a more rapid calcification ${ }^{3}$. Effects of $\mathrm{pH}$ and calcifying medium composition upon calcification quality and degree were observed. The composition and morphology of the calcified samples were analysed by microscopy (scanning electron microscopy - JEOL JXA-840A Electron Probe Microanalyzer) and spectroscopy (EDS).

In order to prepare samples for EDS, substrates were gently washed with Milli-Q water, three times, and then fractured under lower temperatures before freeze-drying. The pieces were then coated with carbon by evaporation in order to allow their observation under electron beam and quantification of $\mathrm{Ca}$ and $\mathrm{P}$ elements by EDS.

\section{Results}

Calcification on chitosan membranes could be observed after a 7-day-period in solutions with $\mathrm{pH}=7.8$ and 7.4. However, no deposition could be observed on samples soaked in solutions with $\mathrm{pH}=7.2$ even after one month.

The morphology of deposits on chitosan membranes was very different from those observed on precipitates obtained by the sol-gel process, without the presence of an organic matrix. The former presented sphere-shaped deposits, also observed by other authors ${ }^{4}$, whilst the latter did not have a well-defined shape. This fact stresses the existence of an influence of the matrix in the process of mineralization (Fig.1a).

The deposits formed in calcium ion excess presented a large spherical shape (c.a. $4 \mu \mathrm{m}$ diameter) whilst the ones formed in phosphate ion excess demonstrated a more irregular shape, a smaller size and better interconnection with the matrix.
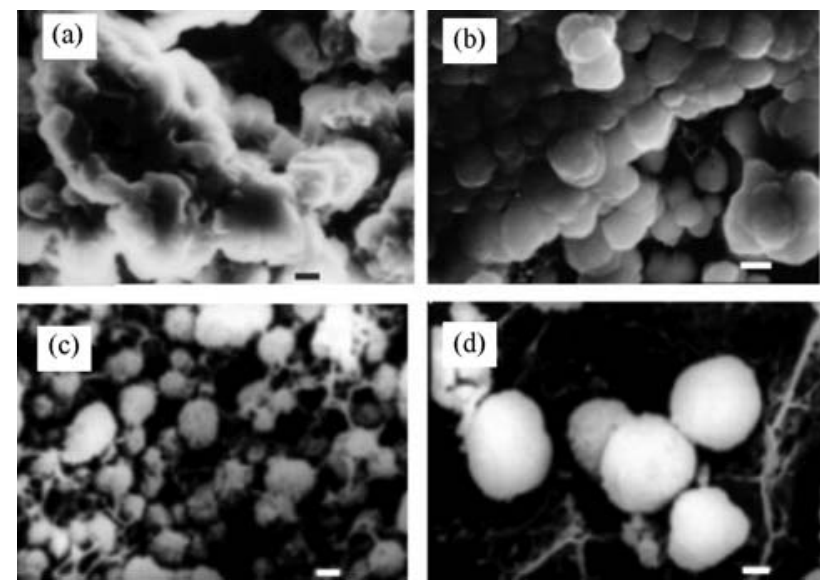

Figure 1. Scanning electron micrographs of (a) sol-gel precipitate; surfaces of porous chitosan membranes (b) calcified at $\mathrm{pH} 7.8$ (c) calcified at $\mathrm{pH} 7.8$ with excess of calcium ions (d) calcified at $\mathrm{pH} 7.8$ with excess of phosphate ions. Bars correspond to $1 \mu \mathrm{m}$.

The median values of at least ten measurements made using the X-ray (EDS) microprobe (used with SEM) are depicted in Table 2. Ca:P ratios have values very close to those found in apatites (Table 2). At pH 7.4, the degree of calcification was too low and the calcification layer was thinner than the depth of the penetration of the X-rays, making it difficult to make reliable measurement of the $\mathrm{Ca}: \mathrm{P}$ ratio by EDS. The Ca:P ratio values were extremely diverse in the excess of calcium or phosphate ion. Findings indicate that the deposition occurs in different manners, since different chemical groups are present in the solution used for the calcification process.

\section{Discussion}

Recently, calcification of bioactive surfaces has been intensively studied. The majority of these studies prioritise

Table 2. Ca:P molar ratios obtained by EDS (average of ten values).

\begin{tabular}{lcccc}
\hline specimen & $\mathrm{pH}$ & Excess of Ca & Excess of P & Ca:P molar ratio \\
\hline Sol-gel precipitate & 7.8 & No & No & 2.01 \\
Porous membrane & 7.8 & No & No & 1.67 \\
Porous membrane & 7.8 & Yes & No & 1.73 \\
Porous membrane & 7.8 & No & Yes & 1.46 \\
Porous membrane & 7.4 & No & No & Not measurable \\
\hline
\end{tabular}

Table 1. Concentration of ions in calcification solutions, in mM.

\begin{tabular}{lccccccccc}
\hline & $\mathrm{pH}$ & $\mathrm{Na}$ & $\mathrm{K}$ & $\mathrm{Ca}$ & $\mathrm{Mg}$ & $\mathrm{Cl}$ & $\mathrm{HCO}_{3}$ & $\mathrm{HPO}_{4}$ & $\mathrm{SO}_{4}$ \\
\hline serum & 7.4 & 142.0 & 5.0 & 2.5 & 1.5 & 103.0 & 27.0 & 1.0 & 0.5 \\
1x CS & 7.8 or 7.4 & 142.5 & 5.0 & 2.5 & 1.5 & 178.0 & 4.5 & 1.0 & 0.5 \\
$1.5 x$ CS & 7.8 or 7.4 & 213.7 & 7.5 & 3.75 & 2.25 & 267.0 & 6.7 & 1.5 & 0.75 \\
Excess of P & 7.8 & 213.7 & 10.5 & 3.75 & 2.25 & 267.0 & 6.7 & 3.0 & 0.75 \\
Excess of Ca & 7.8 & 213.7 & 7.5 & 7.5 & 2.25 & 274.5 & 6.7 & 1.5 & 0.75 \\
\hline
\end{tabular}


studies at lower pHs mainly due to the difficulty in distinguishing the effects of the organic matrix and from spontaneous precipitation in the bulk of the solution. However, the present results show that even in conditions where spontaneous precipitation can occur $(\mathrm{pH} 7.8)$, the matrix has a significant influence of matrix upon deposition. In our case, this can be observed in deposit morphology and composition.

In serum, as well as in the simulated body fluids, the $\mathrm{Ca}: \mathrm{P}$ ratio is 2.5 . The precipitates formed spontaneously present a Ca:P ratio of near to 2 . Conversely, deposits formed on chitosan demonstrate values close to those found in apatites $\left(1.5-1.7^{5}\right)$.

\subsection{Effect of solution $p H$}

From observations of calcification occurrence, it was seen that $\mathrm{pH}$ is a major factor in in vitro calcification. An important parameter that can explain this effect is pKa, which reflects the degree of protonation of chitosan. The protonation of chitosan amino groups can be represented as:

$$
\begin{array}{ll}
\mathrm{Glc}-\mathrm{NH}_{3}{ }^{+}+\mathrm{H}_{2} \mathrm{O} \leftrightarrow \mathrm{Glc}-\mathrm{NH}_{2}+\mathrm{H}_{3} \mathrm{O}^{+} & \alpha \quad \alpha \\
(1-\alpha) & \alpha \\
\mathrm{pKa}=\mathrm{pH}+\log (1-\alpha) / \alpha & \quad \text { (Katchalsky's) }
\end{array}
$$

where $\alpha$ is the fraction of $\mathrm{NH}_{2}$.

Since the pKa of chitosan is in the range of 6.3-7, we can calculate the fraction of amino groups that are not positively charged by a $\mathrm{H}^{+}$(Table 3 ).

As seen in Table 2, at pH 7.8, the majority of the amino groups of chitosan are in the non-protonated form. This condition would allow the interaction of cations through the pair of electrons disposed by nitrogen present in these groups.

Higher $\mathrm{pHs}$ would promote faster calcification due to this effect combined with the fact that calcium compounds are poorly soluble in water solutions when alkalinity increases.

\subsection{Effect of phosphate or calcium ion excess}

Calcification in the excess of calcium or phosphate ions produces different deposits, both in morphology (interconnectivity with matrix) and composition. It is very likely that

Table 3. Fraction of amino groups that are non-charged.

$\alpha$

\begin{tabular}{cccc} 
& $\mathrm{pH}=7.2$ & $\mathrm{pH}=7.4$ & $\mathrm{pH}=7.8$ \\
\hline $\mathrm{pKa}=6.3$ & 0.8882 & 0.9264 & 0.9693 \\
$\mathrm{pKa}=7.0$ & 0.6131 & 0.7153 & 0.8632 \\
\hline
\end{tabular}

the mechanisms of phase separation in both cases are different.

It is known that phase separation can occur by two basic mechanisms: 1) nucleation and growth, where a large distribution of sizes and lower connectivity among nuclei is observed $^{6}$, and 2) Spinodal decomposition, where a large connectivity between two phases is expected.

It is possible that deposition on chitosan under excess of phosphate allows the occurrence of a process more similar to a spinodal decomposition. The deposits under this condition presented a morphology with a large area, indicating that there was a good interconnectivity with the liquid phase near the chitosan surfaces. Under conditions (excess of calcium ions and $\mathrm{pH} 7.8$ ) the deposits demonstrate morphologies similar to the ones observed for nucleation and growth.

Although morphology is not the determinant factor to define the type of separation process, it should be stressed that the mechanisms found in one or other condition are different. Further investigations should be carried out in order to determine with certainty which mechanism is present in each case.

Kokubo ${ }^{7}$ reported that the control of the apatite formed on silica is directly related to the Ca:P ratio in the fluid and further suggested that it is very likely that the ratio Ca:P varies mainly near the frontier of the apatite with fluid. In an interface where there is a lower quantity of available $\mathrm{Ca}$ $(\mathrm{Ca} / \mathrm{P}<1.5)$, this author found a more granulated geometry whilst under converse conditions (excess of $\mathrm{Ca}$ ) he observed a more flaked morphology in precipitates. This morphology is not in agreement with the ones found in the present work, but stresses the idea that the $\mathrm{Ca}: \mathrm{P}$ ratio of the calcification fluid is a very important factor and can determine the manner by which phase separation occurs, and hence, the morphology and composition of phases formed.

\section{Conclusion}

There are two basic theories regarding matrix influence upon mineralization: 1) deposition depends on the matrix chemical nature $\left(\right.$ Kokubo $\left.^{8}\right)$ and 2) in vivo calcification (such as osteo-conduction) is mainly dependent on the micro-mechanics of the surface rather than the chemistry (Davies ${ }^{9}$ ).

In this study, where the substrates were the same, it is clear that the chemical interaction is a very important factor in vitro deposition, and that the composition and $\mathrm{pH}$ of the fluid used for calcification experiments interfered in deposit characteristics.

\section{Acknowledgements}

The authors thanks PRONEX and CNPq for financial support. 


\section{References}

1. Pathak, Y.; Shoen, F.J.; Levy, R.J. Pathologic Calcification of Biomaterials, Biomaterials Science, an Introduction to Materials in Medicine, Ratner, B.D.; Hoffman, A.S.; Schoen, F.J.; Lemons, J.E., eds., Academic Press, California-USA (1996), p. 272-282.

2. Sandford, P.A.. Chitosan: Commercial Uses and Potential Applications, Chitin and Chitosan, SkjaekBraek, G. et al., ed., Elsevier Applied Science, New York, p. 51-69, 1989.

3. Beppu, M.M. Estudo da Calcificação in vitro de Quitosana. PhD Thesis. Unicamp, Brazil.

4. Golomb, G.; Wagner, D. Biomaterials, v. 12, p. 397, 1991.
5. Mucalo, M.R.; Toriyama, M.; Yokogawa, Y.; Suzuki, T.; Kawamoto, Y.; Nagata, F.; Nishizawa, K. J. Mater. Sci. Mater. Medicine, v. 6, p. 409-419, 1995.

6. Utracki, L.A. Polymer Alloys and Blends - Thermodynamics and Rheology. Munich: Hansen Publishers, p. 29-52, 1989.

7.Li, P.; Nakanishi, K.; Kokubo., T.; de Groot, K. Induction and Morphology of Hydroxyapatite, Precipitated from Metaestable Simulated Body Fluids on Sol-Gel Prepared Silica. Biomaterials, v. 14, n. 13, p. 963-968, 1993.

8. Kokubo, T.; Ito, S.; Huang, Z.T.; Hayashi, T.; Sakka, S.; Kitsugi, T.; Yamamuro, T. Journal of Biomed. Mater. Res., v. 24, p. 331-343, 1990.

9. Davies, J.E. Mechanisms of Endosseous Integration, $1^{\text {st }}$ COLAOB, Belo-Horizonte, Brazil, 1998.

FAPESP helped in meeting the publication costs of this article 